\title{
Using carrier-depletion silicon modulators for optical power monitoring
}

\author{
Hui Yu, ${ }^{1, *}$ Dietmar Korn, ${ }^{2}$ Marianna Pantouvaki, ${ }^{3}$ Joris Van Campenhout, ${ }^{3}$ Katarzyna \\ Komorowska, ${ }^{2}$ Peter Verheyen, ${ }^{3}$ Guy Lepage,${ }^{3}$ Philippe Absil, ${ }^{3}$ David Hillerkuss, ${ }^{2}$ Juerg Leuthold, ${ }^{2}$ \\ Roel Baets, ${ }^{1}$ and Wim Bogaerts ${ }^{1}$ \\ ${ }^{I}$ Photonics Research Group, Department of Information Technology, Ghent University-imec, Center for Nano- and \\ Biophotonics (NB Photonics), \\ St.-Pietersnieuwstraat 41, 9000 Gent, Belgium \\ ${ }^{2}$ Institute of Photonics and Quantum Electronics (IPQ) and Institute of Microstructure Technology (IMT), \\ Karlsruhe Institute of Technology(KIT), 76131 Karlsruhe, Germany \\ ${ }^{3}$ imec, Kapeldreef 75, 3001 Leuven, Belgium \\ *Corresponding author: hyu@intec.ugent.be
}

Received Month X, XXXX; revised Month X, XXXX; accepted Month X, XXXX; posted Month X, XXXX (Doc. ID XXXXX); published Month X, XXXX

Defect mediated sub-bandgap absorption is observed in ion-implanted silicon-on-oxide waveguides which experience a rapid thermal annealing at $1075^{\circ} \mathrm{C}$. With this effect, general carrier-depletion silicon modulators exhibit the capability for optical power monitoring. Responsivity is measured to be $22 \mathrm{~mA} / \mathrm{W}$ at $-7.1 \mathrm{~V}$ bias voltage for a $3 \mathrm{~mm}$ long Mach-Zehnder modulator of $2 \times 10^{18} \mathrm{~cm}^{-3}$ doping concentration, and $5.9 \mathrm{~mA} / \mathrm{W}$ at $-10 \mathrm{~V}$ bias voltage for a ring modulator of $1 \times 10^{18} \mathrm{~cm}^{-3}$ doping concentration. The latter is used to demonstrate data detection of up to $35 \mathrm{Gbit} / \mathrm{s}$. (C) 2012 Optical Society of America OCIS Codes: 250.7360, 230.7020, 250.0040

An objective of silicon photonics is to integrate different functionalities, including wavelength multiplexing and demultiplexing, routing, optical emission, modulation, and detection on the silicon-on-oxide (SOI) platform by leveraging the advanced complementary metal-oxidesemiconductor (CMOS) process technology. In case of power monitoring and detection, the $1.12 \mathrm{eV}$ indirect bandgap of defect-free bulk silicon makes it transparent in telecom wavelength bands around $1.31 \mu \mathrm{m}$ and $1.55 \mu \mathrm{m}$. In addition to the incorporation of germanium [1] or III-V semiconductors [2] into a silicon photonic circuit by epitaxial growth or bonding techniques, there are also two intrinsic sub-bandgap absorption mechanisms which can give rise to a photocurrent inside a SOI waveguide: surface-state absorption (SSA) [3] and two photon absorption (TPA) [4]. In order to enhance SSA and TPA, the cross-section of a SOI waveguide is tailored to enlarge the overlap of the optical mode with the waveguide surface [5], also high Q photonic crystal cavities $(\mathrm{PhC})$ are employed to achieve a high optical intensity at a reasonable input power [6,7]. Another mechanism which can generate a photocurrent from infrared light in silicon is the defect mediated sub-bandgap absorption. Crystal defects and associated mid-bandgap states which facilitate the infrared optical absorption are induced by selectively implanting $\mathrm{Si}^{+}$[8-11], $\mathrm{B}^{+}$[12], $\mathrm{He}^{+}$[13], or protons [14] with proper doses and energies.

Generally the photoactive crystal defects include divacancies and interstitial clusters. Divacancies are annealed out of the silicon between 150 and $300{ }^{\circ} \mathrm{C}$, while interstitial clusters are believed to remain stable up to $600{ }^{\circ} \mathrm{C} \quad[10,11]$. J. K. Doylend reported that the photocurrent due to defect mediated absorption decreases by a factor of 100 as the annealing temperature rises from $150{ }^{\circ} \mathrm{C}$ to $400{ }^{\circ} \mathrm{C}$ [15]. The highest annealing temperature reported of these defect dependent devices is $475{ }^{\circ} \mathrm{C}$
[10,11]. It is not clear whether the effect can be observed after annealing at higher temperatures. In this paper we explore the use of defect-state mediated absorption for an integrated power-monitoring function to avoiding increasing the fabrication complexity. While the observed effect is generally much weaker than direct absorption in a Ge or III-V photodiode, the functionality can be implemented relying exclusively on silicon carrierdepletion modulators without adding processing steps.

The carrier-depletion modulator is becoming a preferred solution for electro-optic modulation in silicon as a result of its CMOS compatibility, processing simplicity and high operation speed [16]. With this structure PN junctions are embedded in SOI waveguides by ion implantation. After that, a rapid thermal annealing (RTA) above $1000^{\circ} \mathrm{C}$ activates the dopants and repairs damages in the silicon lattice, without causing much dopant redistribution. Still, there remain some residual crystal defects that can mediate sub-bandgap absorption. After being heated to $1075^{\circ} \mathrm{C}$, a reversed-biased silicon waveguide diode still produces a substantial photocurrent. This means that we can obtain from the carrier-depletion modulator an additional functionality as a power monitor.

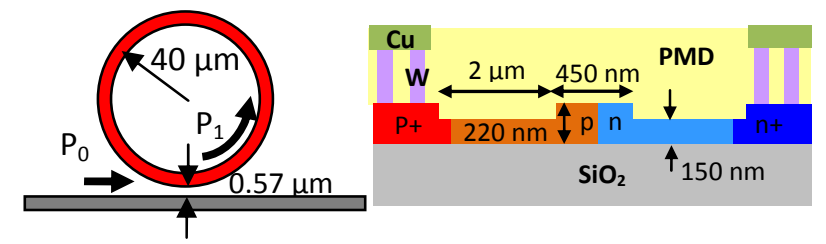

Fig. 1. Schematic diagram of a lateral PN junction based ring modulator.

The modulator used to characterize the photocurrent is a ring resonator with a lateral PN junction as shown in Fig. 1. Fiber grating couplers are used to couple the light in and out. The ring radius and the gap between the ring 
and the bus waveguide are $40 \mu \mathrm{m}$ and $570 \mathrm{~nm}$, respectively. The rib waveguide has a width of $450 \mathrm{~nm}$, a height of $220 \mathrm{~nm}$ and an etch depth of $70 \mathrm{~nm}$. The nominal doping concentration of the PN junction is $1 \times$ $10^{18} / \mathrm{cm}^{3}$, while the contact regions beside the waveguide are heavily doped to $1 \times 10^{20} / \mathrm{cm}^{3}$ to ensure ohm contacts. The device was initially designed and then fabricated by the $200 \mathrm{~mm}$ line of imec for $10 \mathrm{Gbit} / \mathrm{s}$ modulation with a driving voltage of $<1 \mathrm{~V}_{\mathrm{pp}}$ [17]. An elaboration of the processing flow can be found in our previous work [18]. We note that the highest temperature in the whole processing flow was $1075{ }^{\circ} \mathrm{C}$ during the RTA. Unlike the previous works which all require dedicated ion implantation and annealing to introduce defects [8-14], we take advantage of residual defects after RTA, and thus no additional processing steps are needed. This also means no additional absorption (and therefore optical loss) mechanisms are introduced into the modulator.
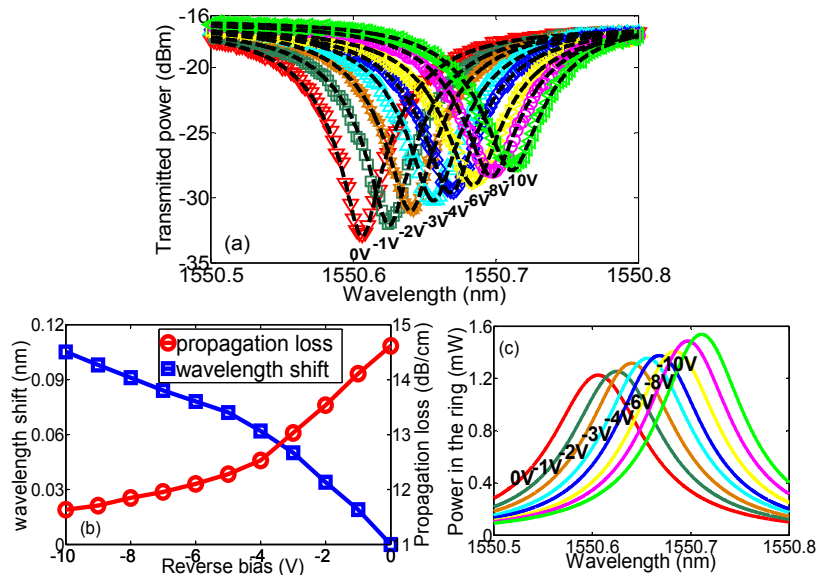

Fig. 2. (a) Transmission spectra of the ring at different biases. (b) Resonance wavelength shift and propagation loss of the ring vs. bias. (c) The power $\mathrm{P}_{1}$ inside the ring for different biases.

Discrete points in Fig. 2(a) show measured transmission spectra of the device at different reverse bias points when the laser power is $0.5 \mathrm{~mW}$. Since the power coupled out from the device at off-resonance wavelengths is $0.02 \mathrm{~mW}(-16.9 \mathrm{dBm})$, we estimate that the on-chip power in the bus waveguide before entering the coupling section of the ring is $P_{0}=0.1 \mathrm{~mW}$ by assuming that the input and the output grating couplers have the same coupling efficiency. We extract the resonance wavelength shift $\Delta \lambda$, cross-coupling coefficient $K$ between the ring and the bus waveguide, and the beam propagation loss of the ring from the transmission spectra by curve fitting. The fitting results are shown as dashed curves in Fig. 2(a). The variation of $\Delta \lambda$ and the loss with the reverse bias are plotted in Fig. 2(b). With good approximation, the value of $K$ is considered fixed at 0.33 . The propagation loss of $14.6 \mathrm{~dB} / \mathrm{cm}$ at $0 \mathrm{~V}$ is quite reasonable for the utilized doping level. The defect mediated absorption should be insignificant compared with the free carrier absorption. With the value of $\Delta \lambda, K$ and the propagation loss, we calculate the power inside the ring after exiting the coupling section ( $\mathrm{P}_{1}$ as shown in Fig. 1). It is depicted in Fig. 2(c) as a function of the wavelength for different reverse biases.

The photocurrent (in DC regime) as a function of wavelength is recorded simultaneously when we measure the spectrum in Fig. 2(a). The result is shown in Fig. 3(a).
Only photocarriers in the carrier-depletion region are drifted by the electrical field and thus contribute to the photocurrent efficiently. Therefore, as the reverse bias increases in Fig. 3(a), the photocurrent is enhanced by the expanded carrier-depletion region. Combining Figs. 2(c) and $3(\mathrm{a})$, we map the relationship between the power $\mathrm{P}_{1}$ in the ring and the photocurrent as shown in Fig. 3(b). Ripples in Figs. 3(a) and 3(b) result from the Fabry-Pérot cavity effect between the two fiber grating couplers. It can be seen that a linear absorption rules the generation of photocurrent. As aforementioned there are two linear absorption mechanisms inside a SOI waveguide: defect mediated absorption and SSA. Before we conclude that the current is caused by residual defects, it is necessary to estimate the contribution from SSA. H. Chen has characterized the SSA induced photocurrent with a PIN diode embedded SOI ring resonator of similar geometry [3]. The on-resonance photocurrent is $25 \mathrm{nA}$ at $-10 \mathrm{~V}$ for an on-chip power of $125 \mu \mathrm{W}$ in the bus waveguide. In contrast, the maximum photocurrent at $-10 \mathrm{~V}$ is $590 \mathrm{nA}$ in Fig. 3 (a) for an on-chip power of $100 \mu \mathrm{W}$ in the bus waveguide. We attribute this $30 \times$ enhancement of the photocurrent to the defect mediated absorption.

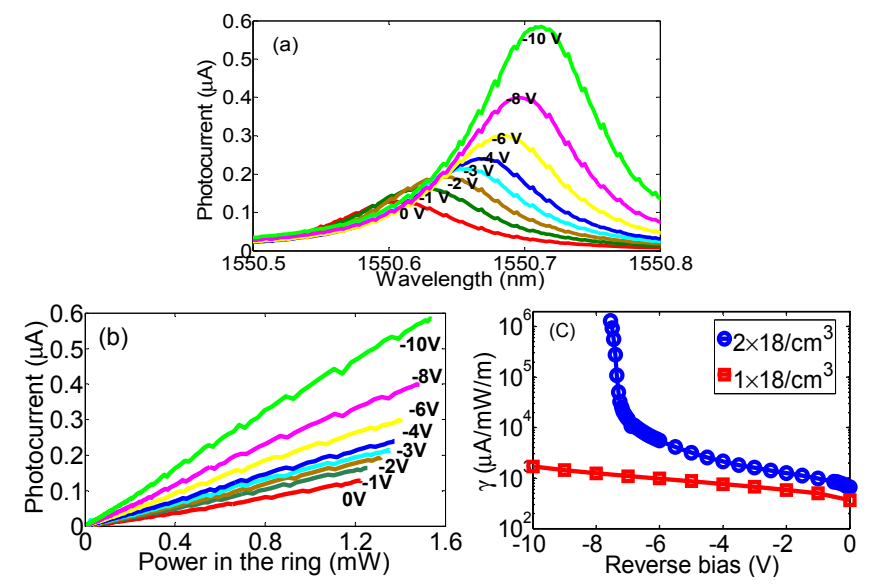

Fig. 3. (a) Spectra of DC photocurrent at different biases. (b) Photocurrent vs. optical power in the ring. (c) Carrier generation efficiency $\gamma$ vs. reverse bias for two doping concentrationes.

The linear photocurrent can be calculated as [12]

$$
i_{\mathrm{ph}}=\frac{1-\exp (-\alpha \mathrm{L})}{\alpha} \mu_{1}
$$

where $P_{1}$ is the power inside the ring after exiting the coupling section, $L$ and $\alpha$ denote the perimeter and the attenuation coefficient of the ring respectively. The carrier generation efficiency $\gamma$ is determined by defect density, photon energy, and distributions of both optical mode and electrical field. The term $\gamma[1-\exp (-\alpha L)] / \alpha$ corresponds to the slope of the curves in Fig. 3(b). Since the propagation loss of the ring is already known in Fig. 2(b), we can deduce the value of $\gamma$ for different reverse biases in Fig. 3(c). In order to explore the relationship between $\gamma$ and the implantation condition, we characterize the photocurrent of a $3 \mathrm{~mm}$ lateral PN junction based MZ modulator (MZM) from another imec run. The device is driven by a coplanar waveguide (CPW) based traveling wave electrode which enables a $>35 \mathrm{~Gb} / \mathrm{s}$ modulation with a pseudorandom binary sequence (PRBS) of $2^{7-1}$ pattern 
length [18]. Ion implantation conditions are the same as the ring modulator except that the dose is twice as high, i.e., $2 \times 10^{18} / \mathrm{cm}^{3}$. Looking at only one arm of the MZM, we speculate that the defect density and the consequent carrier generation efficiency $\gamma$ would scale up similarly. This is confirmed by the comparison in Fig. 3(c). Within the region where both devices are far from diode breakdown, $\gamma$ in one arm the MZM is roughly twice as high as in the ring, e.g., $1264 \mu \mathrm{A} / \mathrm{mW} / \mathrm{m}$ vs. $582 \mu \mathrm{A} / \mathrm{mW} / \mathrm{m}$ at $-2 \mathrm{~V}$. The dramatic increase of $\gamma$ in the blue curve indicates the avalanche multiplication.

The overall responsivity is defined as the photocurrent divided by the optical power coupled into the device [12], i.e., the power in the bus waveguide of the ring and that entering the phase shifters of the MZM. With this definition, the responsivities are $5.9 \mathrm{~mA} / \mathrm{W}$ for the onresonance ring at $-10 \mathrm{~V}$, and $22 \mathrm{~mA} / \mathrm{W}$ for the $3 \mathrm{~mm}$ MZM at $-7.1 \mathrm{~V}$. The corresponding dark currents are $3 \mathrm{nA}$ and $1.8 \mu \mathrm{A}$ for the ring and the MZM respectively. Most of the optical absorption is induced by free carriers rather than by defects, and these absorbed photons do not give rise to any photocurrent.
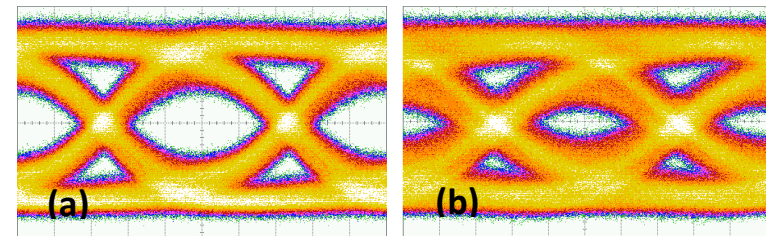

Fig. 4. Eye diagrams of detection at (a) $25 \mathrm{Gbit} / \mathrm{s}$ and (b) $35 \mathrm{Gbit} / \mathrm{s}$.

The response speed of the photocurrent is studied by the eye diagram measurement. We choose the $3 \mathrm{~mm}$ MZM so as to exclude the influence of photon lifetime in the ring. Each phase shifter of the MZ now can behave as a traveling wave detector. The scheme means that the input port of the CPW is terminated with a $50 \mathrm{ohm}$ load, while the output port is connected to an oscilloscope through a RF amplifier. A DC bias is applied on the device by a bias tee. A high bias voltage helps to enhance the responsivity as shown in Fig. 3(c). However, it should not exceed the breakdown voltage to avoid the massive diode breakdown current. Based on the above principle the bias voltage is carefully chosen to be $-7.1 \mathrm{~V}$. A commercially available $40 \mathrm{~Gb} / \mathrm{s} \mathrm{LiNbO}$ modulator is used to encode a PRBS of 27-1 pattern length onto a $1555 \mathrm{~nm}$ optical carrier at different bit rates. The optical power entering each arm of the MZ is estimated to be $9 \mathrm{dBm}$. Clear open eye diagrams indicate that this kind of device has the potential for high speed optical detection. The CPW here is designed for modulation rather than detection. Therefore, the performance can be improved by a dedicated RF optimization.

In conclusion, we confirm the defect mediated subbandgap absorption in ion implanted SOI waveguides (boron as $\mathrm{p}$-dopants and phosphorus as n-dopants) after a RTA of $1075{ }^{\circ} \mathrm{C}$. This means that the widely utilized carrier-depletion based modulator can also be used for optical power monitoring without any additional processing. The extended functionality could have many potential applications especially when a further increase in fabrication complexity and risk can be avoided. For example, carrier-depletion based ring modulators rely on an active tuning with feedback. This usually requires a heater as well as a power monitor to control the operation wavelength. Instead of using a Ge or III-V photodiode for power detection at the drop port of an add-drop ring, we can directly use a small section of the PN junction embedded ring to monitor the power inside. We expect that the responsivity can be enhanced by optimizing the doping pattern and the implantation condition in future.

The authors acknowledge the efforts of imec's 200-mm pline for their contributions to the device fabrication. This work was supported by imec's Core Partner Program, and by the EU-FP7 project SOFI (grant 248609).

\section{References}

1. J. Michel, J. Liu, and L. C. Kimerling, Nature Photon. 4, 527-534 (2010).

2. K. Ohira, K. Kobayashi1, N. Iizuka1, H. Yoshida, M. Ezaki1, H. Uemura, A. Kojima, K. Nakamura, H. Furuyama, and H. Shibata, Opt. Express 18, 15440-15447 (2010).

3. H. Chen, X. Luo, and A. W. Poon, Appl. Phys. Lett. 95, 171111 (2009).

4. H. Chen, and A. W. Poon, Appl. Phys. Lett. 96, 191106 (2010).

5. T. Baehr-Jones, M. Hochberg, and A. Scherer, Opt. Express 16, 1659-1668 (2008).

6. L. D. Haret, X. Checoury, Z. Han, P. Boucaud, S. Combrié, and A. De Rossi, Opt. Express 18, 23965-23972 (2010).

7. T. Tanabe, H. Sumikura, H. Taniyama, A. Shinya, and M. Notomi, Appl. Phys. Lett. 96, 101103 (2010).

8. D. F. Logan, P. Velha, M. Sorel, R. M. De La Rue, A. P. Knights, and P. E. Jessop, IEEE Photon. Technol. Lett. 20, 1530-1532 (2010).

9. M. W. Geis, S. J. Spector, M. E. Grein, R. T. Schulein, J. U. Yoon, D. M. Lennon, S. Deneault, F. Gan, F. X. Kaertner, and T. M. Lyszczarz, IEEE Photon. Technol. Lett. 19, 152154 (2007).

10. M. W. Geis, S. J. Spector, M. E. Grein, R. J. Schulein, J. U. Yoon, D. M. Lennon, C. M. Wynn, S. T. Palmacci, F. Gan, F. X. Käertner, and T. M. Lyszczarz, Opt. Express 15, 16886-16895 (2007).

11. M. W. Geis, S. J. Spector, M. E. Grein, J. U. Yoon, D. M. Lennon, and T. M. Lyszczarz, Opt. Express 17, 5193-5204 (2009).

12. J. K. Doylend, P. E. Jessop, and A. P. Knights, Opt. Express 18, 14671-14678 (2010).

13. Y. Liu, C. W. Cho, W. Y. Cheung, and H. K. Tsang, IEEE Photon. Technol. Lett. 18, 1882-1884 (2006).

14. J. D. B. Bradley, P. E. Jessop, and A. P. Knights, Appl. Phys. Lett. 86, 241103 (2005).

15. J. K. Doylend, A. P. Knights, B. J. Luff, R. Shafiiha, M. Asghari, and R. M. Gwilliam, Electron Lett. 46, 234 (2010).

16. G. T. Reed, G. Mashanovich, F. Y. Gardes, and D. J. Thomson, Nature Photon. 4, 518-526 (2010).

17. M. Pantouvaki, H. Yu, P. Verheyen, G. Lepage, W. Bogaerts, M. Moelants, J. Wouters, D. Radisic, P. Absil, and J. Van Campenhout, in Proceedings of IEEE Conference on Optical Interconncects (IEEE, 2012), pp. 44-45.

18. H. Yu, M. Pantouvaki, J. Van Campenhout, D. Korn, K. Komorowska, P. Dumon, Y. Li, P. Verheyen, P. Absil, L. Alloatti, D. Hillerkuss, J. Leuthold, R. Baets, and W. Bogaerts, Opt. Express 20, 12926-12938 (2012). 
1. J. Michel, J. Liu, L. C. Kimerling, "High-performance Ge-on-Si photodetectors," Nature Photon. 4, 527-534 (2010).

2. K. Ohira, K. Kobayashi, N. Iizuka, H. Yoshida, M. Ezaki, H. Uemura, A. Kojima, K. Nakamura, H. Furuyama, and H. Shibata, "On-chip optical interconnection by using integrated III-V laser diode and photodetector with silicon waveguide," Opt. Express 18, 15440-15447 (2010).

3. H. Chen, X. Luo, and A. W. Poon, "Cavity-enhanced photocurrent generation by $1.55 \mu \mathrm{m}$ wavelengths linear absorption in a p-i-n diode embedded silicon microring resonator," Appl. Phys. Lett. 95, 171111 (2009).

4. H. Chen, A. W. Poon, "Two-photon absorption photocurrent in p-i-n diode embedded silicon microdisk resonators," Appl. Phys. Lett. 96, 191106 (2010).

5. T. Baehr-Jones, M. Hochberg, and A. Scherer, "Photodetection in silicon beyond the band edge with surface states," Opt. Express 16, 1659-1668 (2008).

6. L. D. Haret, X. Checoury, Z. Han, P. Boucaud, S. Combrié, and A. De Rossi, "All-silicon photonic crystal photoconductor on silicon-on-insulator at telecom wavelength,” Opt. Express 18, 23965-23972 (2010).

7. T. Tanabe, H. Sumikura, H. Taniyama, A. Shinya, and M. Notomi, "All-silicon sub-Gb/s telecom detector with low dark current and high quantum efficiency on chip," Appl. Phys. Lett. 96, 101103 (2010).

8. D. F. Logan, P. Velha, M. Sorel, R. M. De La Rue, A. P. Knights, and P. E. Jessop, "Defect-enhanced silicon-oninsulator waveguide resonant photodetector with high sensitivity at 1. $55 \mu \mathrm{m}$," IEEE Photon. Technol. Lett. 20, 1530-1532 (2010).

9. M. W. Geis, S. J. Spector, M. E. Grein, R. T. Schulein, J. U. Yoon, D. M. Lennon, S. Deneault, F. Gan, F. X. Kaertner, and T. M. Lyszczarz, "CMOS-compatible all-Si high-speed waveguide photodiodes with high responsivity in near-infrared communication band," IEEE Photon. Technol. Lett. 19, 152-154 (2007).

10. M. W. Geis, S. J. Spector, M. E. Grein, R. J. Schulein, J. U. Yoon, D. M. Lennon, C. M. Wynn, S. T. Palmacci, F. Gan, F. X. Käertner, and T. M. Lyszczarz, "All silicon infrared photodiodes: photo response and effects of processing temperature," Opt. Express 15, 16886-16895 (2007).

11. M. W. Geis, S. J. Spector, M. E. Grein, J. U. Yoon, D. M. Lennon, and T. M. Lyszczarz, "Silicon waveguide infrared photodiodes with $>35 \mathrm{GHz}$ bandwidth and phototransistors with 50 AW-1 response," Opt. Express 17, 5193-5204 (2009).

12. J. K. Doylend, P. E. Jessop, and A. P. Knights, "Silicon photonic resonator-enhanced defect-mediated photodiode for sub-bandgap detection," Opt. Express 18, 14671-14678 (2010).

13. Y. Liu, C. W. Cho, W. Y. Cheung, and H. K. Tsang, "In-line channel power monitor based on helium ion implantation in silicon-on-insulator waveguides," IEEE Photon. Technol. Lett. 18, 1882-1884 (2006).

14. J. D. B. Bradley, P. E. Jessop, and A. P. Knights, "Silicon waveguide-integrated optical power monitor with enhanced sensitivity at $1550 \mathrm{~nm}$," Appl. Phys. Lett. 86, 241103 (2005).
15. J. K. Doylend, A. P. Knights, B. J. Luff, R. Shafiiha, M. Asghari, and R. M. Gwilliam, "Modifying functionality of variable optical attenuator to signal monitoring through defect engineering," Electron Lett. 46, 234 (2010).

16. G. T. Reed, G. Mashanovich, F. Y. Gardes, and D. J. Thomson, "Silicon optical modulators," Nature Photon. 4, 518-526 (2010).

17. M. Pantouvaki, H. Yu, P. Verheyen, G. Lepage, W. Bogaerts, M. Moelants, J. Wouters, D. Radisic, P. Absil, and J. Van Campenhout, "Lateral versus Interdigitated Diode Design for $10 \mathrm{~Gb} / \mathrm{s}$ Low- Voltage Low Low-Loss Silicon Ring Modulators," in Proceedings of IEEE Conference on Optical Interconncects (IEEE, 2012), pp. 44-45.

18. H. Yu, M. Pantouvaki, J. Van Campenhout, D. Korn, K. Komorowska, P. Dumon, Y. Li, P. Verheyen, P. Absil, L. Alloatti, D. Hillerkuss, J. Leuthold, R. Baets, and W. Bogaerts, "Performance tradeoff between lateral and interdigitated doping patterns for high speed carrierdepletion based silicon modulators," Opt. Express 20, 12926-12938 (2012). 